\title{
Clinical significance of abnormal chest radiographic findings for acute kidney injury in patients with scrub typhus
}

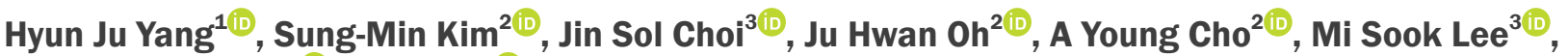 \\ Kwang Young Lee ${ }^{2(\mathbb{D}}$, In 0 Sun $^{2}$ (i) \\ ${ }^{1}$ Division of Pulmonology, Department of Internal Medicine, ${ }^{2}$ Division of Nephrology, Department of Internal Medicine, ${ }^{3}$ Department \\ of Radiology, Presbyterian Medical Center, Jeonju, Republic of Korea
}

\begin{abstract}
Background: Abnormal chest radiographs are frequently encountered in patients with scrub typhus. This study aimed to investigate whether chest radiography on admission is significant as a predictive factor for acute kidney injury (AKI) in patients with scrub typhus.

Methods: From 2010 to 2016, 467 patients were diagnosed with scrub typhus in our hospital. We divided the patients into two groups: normal chest radiograph (NCR) and abnormal chest radiograph (AbNCR), based on chest radiography findings. The incidence, clinical characteristics, and severity of AKI were compared between AKI and nonAKI groups according to the RIFLE classification.

Results: Of the 467 patients, 96 (20.6\%) constituted the AbNCR group. Compared with NCR patients, AbNCR patients were older $(71 \pm 11$ vs. $62 \pm 13$ years, $P<0.001)$ and had higher total leukocyte counts $\left(9.43 \times 10^{3} / \mathrm{mL}\right.$ vs. $6.98 \times$ $\left.10^{3} / \mathrm{mL}, P<0.001\right)$. The AbNCR group had significantly longer duration of hospital stay $(8.9 \pm 5.5 \mathrm{vs} .6 .3 \pm 2.8$ days, $P$ $<0.001)$ and higher incidence of AKI (46.9\% vs. $15.1 \%, P<0.001)$. The common abnormal chest radiographic findings were pulmonary abnormalities, such as pulmonary congestion and pleural effusion. The overall AKI incidence was $21.6 \%$, of which $12.4 \%, 7.9 \%$, and $1.3 \%$ cases were classified as risk, injury, and failure, respectively. In a multivariable logistic regression analysis for association with AKI, old age, presence of chronic kidney disease or hypertension, leukocytosis, hypoalbuminemia, and chest radiographic abnormalities on admission were significant predictors of AKI. Conclusion: Chest radiographic abnormalities on admission were independently associated with AKI in patients with scrub typhus.
\end{abstract}

Keywords: Acute kidney injury, Chronic kidney disease, Radiography, Scrub typhus

Received December 6, 2019; Revised February 6, 2020;

Accepted February 6, 2020

Edited by Eun Hui Bae, Chonnam National University, Gwangju, Republic of Korea

Correspondence: In O Sun

Division of Nephrology, Department of Internal Medicine, Presbyterian Medical Center, 365 Seowon-ro, Wansan-gu, Jeonju 54987, Republic of Korea. E-mail: inogood@catholic.ac.kr

Copyright (c) 2020 by The Korean Society of Nephrology

(a) This is an open-access article distributed under the terms of the Creative Commons Attribution Non-Commercial License (http://creativecommons. org/licenses/by-nc-nd/4.0/), which permits unrestricted non-commercial use, distribution, and reproduction in any medium, provided the original work is properly cited.

\section{Introduction}

Scrub typhus is an acute febrile disease caused by infection with Orientia tsutsugamushi, and is characterized by focal and disseminated vasculitis that can involve many vital organs [1-3]. The spectrum of clinical severity for scrub typhus is vast, ranging from mild infection to a severe and fatal course [1-3]. Depending on the organs involved, patients may present with pneumonitis, acute respiratory distress syndrome, meningitis, myocarditis, acute kidney injury (AKI), or even multiple organ failure 
[3-5].

The incidence of AKI among scrub typhus cases has been reported to range from $21 \%$ to $43 \%$ [6-8]. Old age, comorbidities, and biomarkers, including serum neutrophil gelatinase-associated lipocalin, are associated with AKI development $[8,9]$. Also, pulmonary involvement is a well-known complication of scrub typhus and may vary in severity from mild bronchitis to severe acute respiratory distress syndrome requiring mechanical ventilation. Chest abnormalities associated with scrub typhus are interstitial pneumonia, cardiomegaly, pulmonary edema, pleural effusion, and focal atelectasis [10-13]. However, there are limited data about the association between chest radiographic abnormalities and AKI in patients with scrub typhus.

Therefore, we investigated the association between chest radiographic abnormalities and AKI in patients with scrub typhus.

\section{Methods}

\section{Patient selection}

Between 2010 and 2016, we reviewed 501 patients who presented with acute febrile illness and rash and were diagnosed with scrub typhus, which was confirmed by positive immunoglobulin M enzyme-linked immunosorbent assay (IgM ELISA; InBios International Inc., Seattle, WA, USA). Patients who were transferred to another hospital during treatment or had concomitant infections like leptospirosis, malaria, or dengue fever were excluded from the study. We also excluded patients who were not followed-up during complete renal function recovery or for at least three months after discharge. Patients who were transferred from a local hospital or who underwent anteroposterior (AP) chest X-ray on admission were also excluded from this study. Therefore, a total of 467 patients were enrolled in this study and were divided into two groups, normal chest radiograph (NCR) or abnormal chest radiograph (AbNCR), according to the chest radiography findings on admission. This study was approved by the Institutional Review Board of the Presbyterian Medical Center, Jeonju, South Korea (approval number: 201911-046).

\section{Clinical and laboratory information}

Patients' baseline demographic, clinical, and laboratory data were reviewed upon admission and during the follow-up period. AKI was defined based on the RIFLE (acronym indicating Risk of renal dysfunction; Injury to the kidney; Failure of kidney function, Loss of kidney function and End-stage kidney disease) criteria, and patients were categorized into risk (R), injury (I) or failure (F) categories [14]. Estimated glomerular filtration rate (eGFR) was estimated using the abbreviated Modification of Diet in Renal Disease (MDRD) equation [15]. When baseline serum creatinine was not available, it was calculated using the standard four-variable MDRD formula, assuming an eGFR of $75 \mathrm{~mL} / \mathrm{min} / 1.73 \mathrm{~m}^{2}$. RIFLE category was assigned according to each patient's worst values for serum creatinine, eGFR, and urine output criteria. Renal replacement therapy was initiated using standard indications. Chest radiograph images were reviewed by two radiologists and a final decision was reached by consensus. Plain chest radiographs were used to identify the
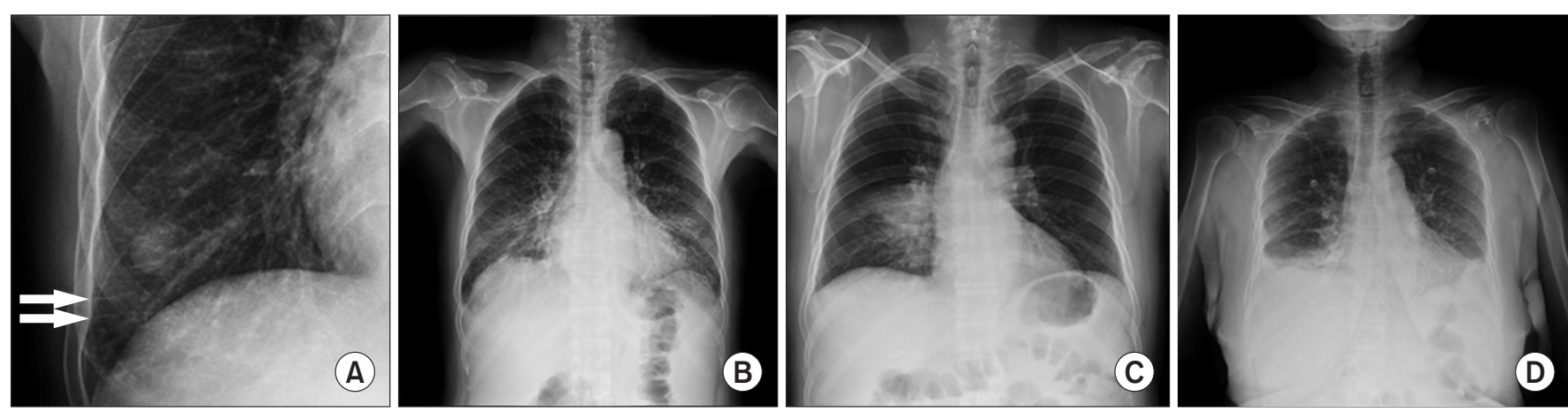

Figure 1. Abnormal chest radiographic findings in scrub typhus patients include septal lines (arrows) (A), pulmonary congestion (B), patchy consolidation (C), and pleural effusion (D). 
following abnormalities: septal lines, pulmonary congestion, patchy consolidation, and focal atelectasis (Fig. 1). The presence of fissure thickening, pleural effusion, or cardiomegaly was also recorded. Previous abnormalities such as sequalae from pulmonary tuberculosis were excluded from this study. All data are presented as means \pm standard deviations unless otherwise specified. Patient baseline characteristics from two groups were compared using the $t$ test, chi-square test, or Fisher exact test, as appropriate. Clinically relevant parameters or the variables that were significantly associated with AKI from univariable analyses were included in the multivariable logistic regression analysis. A $P$ value $<0.05$ was considered statistically significant. Statistical analyses were carried out using IBM SPSS version 22.0 (IBM Corp., Armonk, NY, USA).

\section{Results}

\section{Comparison of clinical characteristics between the NCR and AbNCR groups}

Compared with NCR patients, AbNCR patients were older (71 \pm 11 vs. $62 \pm 13$ years, $P<0.001)$ and more fre-

Table 1. Comparison of baseline characteristics between normal and abnormal chest radiograph groups

\begin{tabular}{lccc}
\hline \multicolumn{1}{c}{ Characteristic } & $\begin{array}{c}\text { NCR } \\
(\mathrm{n}=371)\end{array}$ & $\begin{array}{c}\text { AbNCR } \\
(\mathrm{n}=96)\end{array}$ & P value \\
\hline Age $(\mathrm{yr})$ & $62 \pm 13$ & $71 \pm 11$ & $<0.001$ \\
Male & $136(36.7)$ & $31(32.3)$ & $\mathrm{NS}$ \\
Duration of hospital stay $(\mathrm{d})$ & $6.3 \pm 2.8$ & $8.9 \pm 5.5$ & $<0.001$ \\
Diabetes & $67(18.1)$ & $18(18.8)$ & $\mathrm{NS}$ \\
Hypertension & $134(36.1)$ & $42(43.8)$ & $\mathrm{NS}$ \\
Chronic kidney disease & $11(3.0)$ & $8(8.3)$ & 0.025 \\
Hemoglobin $(\mathrm{g} / \mathrm{dL})$ & $12.9 \pm 1.60$ & $11.9 \pm 1.65$ & $<0.001$ \\
Leukocyte $\left(\times 10^{3} / \mathrm{mL}\right)$ & $6.98 \pm 4.67$ & $9.43 \pm 3.95$ & $<0.001$ \\
Platelet count $\left(\times 10^{3} / \mathrm{mL}\right)$ & $139 \pm 58$ & $130 \pm 51$ & $\mathrm{NS}$ \\
Total bilirubin $(\mathrm{mg} / \mathrm{dL})$ & $0.73 \pm 0.41$ & $0.85 \pm 0.82$ & $\mathrm{NS}$ \\
Serum albumin $(\mathrm{g} / \mathrm{dL})$ & $3.72 \pm 0.48$ & $3.11 \pm 0.50$ & $<0.001$ \\
Serum ALT $(\mathrm{IU} / \mathrm{L})$ & $80 \pm 91$ & $98 \pm 130$ & $\mathrm{NS}$ \\
Creatinine $(\mathrm{mg} / \mathrm{dL})$ & $0.98 \pm 0.39$ & $1.11 \pm 0.59$ & 0.010 \\
eGFR $\left(\mathrm{mL} / \mathrm{min} / 1.73 \mathrm{~m}^{2}\right)$ & $71 \pm 24$ & $59 \pm 28$ & $<0.001$ \\
AKI & $56(15.1)$ & $45(46.9)$ & $<0.001$ \\
\hline
\end{tabular}

Data are presented as number (\%) or mean \pm standard deviation.

AbNCR, abnormal chest radiograph; AKI, acute kidney injury; ALT, alanine aminotransferase concentrations; eGFR, estimated glomerular filtration rate; NCR, normal chest radiograph; NS, not significant. quently had a history of chronic kidney disease ( $8.3 \%$ vs. $3.0 \%, P=0.025$ ) (Table 1). The duration of hospital stay was longer for AbNCR patients than NCR patients ( $8.9 \pm 5.5$ vs. $6.3 \pm 2.8$ days, $P<0.001)$. AbNCR patients had higher total leukocyte counts $\left(9.43 \times 10^{3} / \mathrm{mL}\right.$ vs. $6.98 \times 10^{3} / \mathrm{mL}$, $P<0.001)$ and lower serum albumin levels than NCR patients $(3.11 \pm 0.50$ vs. $3.72 \pm 0.48 \mathrm{~g} / \mathrm{dL}, P<0.001)$. The incidence of AKI was higher in the AbNCR group than in the NCR group $(46.9 \%$ vs. $15.1 \%, P<0.001)$. Plasma alanine aminotransferase concentrations (ALT) were not different between the two groups.

\section{Comparison of clinical characteristics between the AKI and non-AKI groups}

Compared with non-AKI patients, AKI patients were older $(71 \pm 10$ vs. $62 \pm 13$ years, $P<0.001)$ and were associated with a higher incidence of comorbidities, such as hypertension, diabetes, and chronic kidney disease (Table 2). AKI patients had worse renal function and were associated with a higher frequency of AbNCR (45\% vs. $14 \%, P<0.001)$ on admission. Inflammatory markers,

Table 2. Comparison of baseline characteristics between AKI and non-AKI groups

\begin{tabular}{lccc}
\hline \multicolumn{1}{c}{ Characteristic } & $\begin{array}{c}\text { Non-AKI } \\
(\mathrm{n}=366)\end{array}$ & $\begin{array}{c}\text { AKI } \\
(\mathrm{n}=101)\end{array}$ & $P$ value \\
\hline Age $(\mathrm{yr})$ & $62 \pm 13$ & $71 \pm 10$ & $<0.001$ \\
Male & $128(35.0)$ & $39(38.6)$ & $\mathrm{NS}$ \\
Duration of hospital stay (d) & $6.3 \pm 2.8$ & $8.7 \pm 5.4$ & $<0.001$ \\
Diabetes & $56(15.3)$ & $29(28.7)$ & 0.002 \\
Hypertension & $111(30.3)$ & $65(64.4)$ & $<0.001$ \\
Chronic kidney disease & $2(0.5)$ & $17(16.8)$ & $<0.001$ \\
Hemoglobin $(\mathrm{g} / \mathrm{dL})$ & $12.93 \pm 1.57$ & $12.0 \pm 1.80$ & $<0.001$ \\
Leukocyte $\left(\times 10^{3} / \mathrm{mL}\right)$ & $6.78 \pm 4.43$ & $10.01 \pm 4.51$ & $<0.001$ \\
Platelet count $\left(\times 10^{3} / \mathrm{mL}\right)$ & $142 \pm 56$ & $121 \pm 54$ & $<0.001$ \\
Total bilirubin $(\mathrm{mg} / \mathrm{dL})$ & $0.71 \pm 0.37$ & $0.90 \pm 0.86$ & $\mathrm{NS}$ \\
Serum albumin $(\mathrm{g} / \mathrm{dL})$ & $3.70 \pm 0.49$ & $3.22 \pm 0.55$ & $<0.001$ \\
Serum ALT (IU/L) & $86 \pm 102$ & $76 \pm 93$ & $\mathrm{NS}$ \\
Creatinine $(\mathrm{mg} / \mathrm{dL})$ & $0.89 \pm 0.22$ & $1.42 \pm 0.71$ & $<0.001$ \\
eGFR (mL/min/1.73 $\left.{ }^{2}\right)$ & $77 \pm 21$ & $38 \pm 16$ & $<0.001$ \\
Abnormal chest radiographic & $51(13.9)$ & $45(44.6)$ & $<0.001$ \\
$\quad$ findings on admission & & & \\
Abnormal chest radiographic & $74(20.2)$ & $48(47.5)$ & $<0.001$ \\
findings during hospitalization & & & \\
\hline Data are presen & & & \\
\hline
\end{tabular}

Data are presented as mean \pm standard deviation or number (\%).

AKI, acute kidney injury; ALT, alanine aminotransferase concentrations; eGFR, estimated glomerular filtration rate; NS, not significant. 
Yang, et al. Chest radiographs in scrub typhus

Table 3. Univariable and multivariable analyses for association with acute kidney injury

\begin{tabular}{|c|c|c|c|c|}
\hline & \multicolumn{2}{|c|}{ Univariable } & \multicolumn{2}{|c|}{ Multivariable } \\
\hline & HR $(95 \% \mathrm{Cl})$ & $P$ value & $\mathrm{HR}(95 \% \mathrm{Cl})$ & $P$ value \\
\hline Age & 1.063 (1.041-1.086) & $<0.001$ & $1.027(1.001-1.054)$ & 0.040 \\
\hline DM & $2.230(1.330-3.737)$ & $<0.001$ & $1.644(0.829-3.263)$ & 0.155 \\
\hline Hypertension & $4.148(2.608-6.598)$ & $<0.001$ & $2.508(1.400-4.495)$ & 0.002 \\
\hline CKD & 36.833 (8.349-162.495) & $<0.001$ & $24.707(5.087-119.990)$ & $<0.001$ \\
\hline CRA on admission & 4.963 (3.036-8.113) & $<0.001$ & 2.158 (1.140-4.084) & 0.018 \\
\hline Hemoglobin & $0.703(0.609-0.812)$ & 0.703 & $0.989(0.817-1.197)$ & 0.911 \\
\hline Leukocytosis & 1.165 (1.103-1.230) & $<0.001$ & $1.110(1.051-1.172)$ & $<0.001$ \\
\hline Hypoalbuminemia & 6.065 (3.735-9.849) & $<0.001$ & $2.722(1.449-5.113)$ & 0.002 \\
\hline
\end{tabular}

$\mathrm{Cl}$, confidence interval; $\mathrm{CKD}$, chronic kidney disease; CRA, chest radiograph abnormality; DM, diabetes mellitus; HR, hazard ratio.

such as total leukocyte count $\left(10.01 \times 10^{3} / \mathrm{mL}\right.$ vs. $6.78 \times$ $\left.10^{3} / \mathrm{mL}, P<0.001\right)$, were higher in AKI patients than in patients who did not develop AKI. Plasma ALT concentrations were not different between the two groups.

\section{Clinical characteristics of AKI in patients with scrub typhus}

Applying the RIFLE criteria, 58 (57.4\%), 37 (36.6\%), and $6(5.9 \%)$ patients were classified into the $\mathrm{R}, \mathrm{I}$, and $\mathrm{F}$ categories, respectively. Prior to admission, 100 patients had AKI and six patients developed AKI during hospitalization. All except one patient recovered within three months without renal replacement therapy. Univariable analyses identified several significant factors associated with AKI: age, presence of comorbidities, such as hypertension, diabetes, or chronic kidney disease, occurrence of at least one episode of hypotension, thrombocytopenia, total leukocyte count, hypoalbuminemia, or chest radiographic abnormalities on admission. After adjusting for these factors in multivariable logistic regression analysis, age, chronic kidney disease, hypertension, hypoalbuminemia, leukocytosis, and chest radiographic abnormalities on admission were significant predictors of AKI (Table 3).

\section{Chest radiographic findings in scrub typhus cases}

Chest radiographs obtained on admission showed abnormalities in 20.6\% (96/467) of patients. During hospitalization, 122 (26.1\%) patients developed chest radiographic abnormalities (Table 4). The most common findings were pulmonary abnormalities $(88.5 \%, 108 / 122)$, of which pulmonary congestion $(36.9 \%, 45 / 122)$ and patchy consolidation $(32.8 \%, 40 / 122)$ were common findings. Of
Table 4. Abnormal chest radiographic findings in scrub typhus patients during hospitalization $(n=122)$

\begin{tabular}{lc}
\hline \multicolumn{1}{c}{ Findings } & Number (\%) \\
\hline Pulmonary abnormalities & $108(88.5)$ \\
Septal lines & $18(14.8)$ \\
Increased pulmonary vascularity & $5(4.1)$ \\
Pulmonary congestion & $45(36.9)$ \\
Patchy consolidation & $40(32.8)$ \\
Focal atelectasis & $12(9.8)$ \\
Pleural effusion & $65(53.3)$ \\
Fissure thickening & $5(4.1)$ \\
Cardiac enlargement & $7(5.7)$ \\
\hline
\end{tabular}

the 122 patients with AbNCRs, 65 (53.3\%) showed pleural effusions.

\section{Discussion}

Patients with AKI had a higher frequency of chest radiographic abnormalities than those without AKI, and the presence of chest radiographic abnormalities on admission was a significant predictor of AKI in scrub typhus patients. Therefore, our findings provide a rationale for checking chest radiographs on admission as a tool for predicting AKI in patients with scrub typhus.

Scrub typhus is endemic to geographically distinct regions, including Asia-Pacific countries. Common clinical manifestations of scrub typhus include fever, eschar, maculopapular rash, headache, myalgia, cough, nausea, vomiting, conjunctival injection, and dizziness [16]. It can cause multiorgan complications, including AKI, which has an associated incidence of $21 \%$ to $43 \%$ [4-6]. In this study, AKI incidence was $21.6 \%$, which was similar to previous results. Additionally, old age, chronic kidney 
disease, hypertension, leukocytosis, hypoalbuminemia, and chest radiographic abnormalities on admission were significant predictors of AKI. Although there have been reports of chest radiographic findings in patients with scrub typhus, there is a paucity of literature about the clinical significance of chest radiography findings for AKI in patients with scrub typhus on admission. To our knowledge, this is the first study to show a correlation between renal and pulmonary involvement in patients with scrub typhus.

Although the exact pathophysiologic mechanisms are poorly understood, the underlying pathologic process contributing to pulmonary involvement in patients with scrub typhus is interstitial pneumonia, with or without vasculitis [12]. Hwang et al [17] reported that the lungs of mice infected with rickettsia showed marked dilatation and congestion of the septal capillaries, extravasation of red blood cells into the alveoli, and septal widening by lymphocytes and histiocytes. The incidence of chest radiographic abnormalities in patients with scrub typhus varies from $42 \%$ to $72 \%$, with diverse radiologic findings, including pulmonary edema with air-space consolidation, interstitial pneumonia, cardiomegaly, and pleural effusion [12,18-20], of which bilateral diffuse areas of reticulonodular opacity and septal lines are the most common findings [12,13]. In our study, chest radiographic abnormality incidences on admission and during hospitalization were $20.6 \%$ and $26.1 \%$, respectively, which were lower than previously reported, which may be due to referral bias. Previous studies have reported chest radiographic abnormality incidences from tertiary care centers; thus, those data were obtained from severe scrub typhus cases. In contrast, our hospital is a secondary care center and is located adjacent to a rural area. Thus, many patients may visit our hospital when they have an earlystage infection that has not progressed to severe disease. Indeed, Hwang et al [21] reported that the AKI incidence among scrub typhus cases in a tertiary care center was $35.9 \%$.

Although severe scrub typhus is not clearly defined, severity is usually determined by organ involvement $[22,23]$. Patients with severe scrub typhus can experience multiple simultaneous organ failure because the central pathological mechanism of scrub typhus is widespread vasculitis or perivasculitis of multiple organs. Thus, Song et al [13] reported that patients with interstitial pneu- monia tended to have a higher incidence of acute renal failure than patients without interstitial pneumonia. We investigated the correlation between renal and pulmonary involvement. Fifty patients $(10.7 \%)$ experienced simultaneous pulmonary and renal involvement during hospitalization. Furthermore, chest radiographic abnormalities on admission were closely associated with AKI in scrub typhus patients. Therefore, it is essential to check chest radiographs for patients with scrub typhus to monitor renal function. Besides chest radiographic abnormalities on admission, old age, presence of chronic kidney disease, hypertension, leukocytosis, and hypoalbuminemia were significant predictors of AKI, which is consistent with previous findings. Thus, it is necessary to closely monitor renal function in scrub typhus patients who present with these risk factors.

Our study has some limitations. First, this was a retrospective study focused on a single center. Second, we did not enroll patients that were treated in an outpatient clinic. Thus, it is possible that we excluded patients with mild scrub typhus cases. A large prospective randomized controlled study is warranted to investigate the clinical significance of chest radiographic abnormalities in patients with scrub typhus.

The incidence of scrub typhus-associated AKI in our study was $21.6 \%$. The incidences of chest radiographic abnormalities on admission and during hospitalization were $20.6 \%$ and $26.1 \%$, respectively. Of the chest radiographic abnormalities, pulmonary abnormalities were most commonly observed, and the presence of chest radiographic abnormalities on admission was a risk factor for predicting AKI in scrub typhus patients. Therefore, performing chest radiograph on admission may aid in predicting AKI in these patients.

\section{Conflicts of interest}

All authors have no conflicts of interest to declare.

\section{Authors' contributions}

Hyun Ju Yang and In O Sun designed the study. SungMin Kim, Jin Sol Choi, Ju Hwan Oh, and Mi Sook Lee participated in the data collection and performed the statistical analysis. In O Sun and Kwang Young Lee wrote the manuscript. A Young Cho and In O Sun provided in- 
tellectual content of the critical importance to the work and technical support. All authors read and approved the final manuscript.

\section{References}

[1] Varghese GM, Abraham OC, Mathai D, et al. Scrub typhus among hospitalised patients with febrile illness in South India: magnitude and clinical predictors. J Infect 2006;52:5660.

[2] Mahajan SK. Scrub typhus. J Assoc Physicians India 2005; 53:954-958.

[3] Mandell GL, Bennett JR, Dolin R. Mandell, Douglas, and Bennett's principles and practice of infectious diseases. 7th ed. Philadelphia: Churchill Livingstone; 2010. p. 2529-2530.

[4] Silpapojakul K, Ukkachoke C, Krisanapan S, Silpapojakul K. Rickettsial meningitis and encephalitis. Arch Intern Med 1991;151:1753-1757.

[5] Hsu GJ, Young T, Peng MY, Chang FY, Chou MY, Sheu LF. Acute renal failure associated with scrub typhus: report of a case. J Formos Med Assoc 1993;92:475-477.

[6] Basu G, Chrispal A, Boorugu H, et al. Acute kidney injury in tropical acute febrile illness in a tertiary care centre--RIFLE criteria validation. Nephrol Dial Transplant 2011;26:524531.

[7] Attur RP, Kuppasamy S, Bairy M, et al. Acute kidney injury in scrub typhus. Clin Exp Nephrol 2013;17:725-729.

[8] Sun IO, Kim MC, Park JW, et al. Clinical characteristics of acute kidney injury in patients with scrub typhus--RIFLE criteria validation. J Infect Chemother 2014;20:93-96.

[9] Sun IO, Shin SH, Cho AY, Yoon HJ, Chang MY, Lee KY. Clinical significance of NGAL and KIM-1 for acute kidney injury in patients with scrub typhus. PLoS One 2017;12:e0175890.

[10] Tsay RW, Chang FY. Serious complications in scrub typhus. J Microbiol Immunol Infect 1998;31:240-244.

[11] Chi WC, Huang JJ, Sung JM, Lan RR, Ko WC, Chen FF. Scrub typhus associated with multiorgan failure: a case report. Scand J Infect Dis 1997;29:634-635.

[12] Choi YH, Kim SJ, Lee JY, Pai HJ, Lee KY, Lee YS. Scrub typhus: radiological and clinical findings. Clin Radiol 2000; 55:140-144.

[13] Song SW, Kim KT, Ku YM, et al. Clinical role of interstitial pneumonia in patients with scrub typhus: a possible marker of disease severity. J Korean Med Sci 2004;19:668-673.

[14] Bellomo R, Ronco C, Kellum JA, Mehta RL, Palevsky P; Acute Dialysis Quality Initiative workgroup. Acute renal failure - definition, outcome measures, animal models, fluid therapy and information technology needs: the Second International Consensus Conference of the Acute Dialysis Quality Initiative (ADQI) Group. Crit Care 2004;8:R204R212.

[15] Levey AS, Coresh J, Greene T, et al.; Chronic Kidney Disease Epidemiology Collaboration. Expressing the Modification of Diet in Renal Disease Study equation for estimating glomerular filtration rate with standardized serum creatinine values. Clin Chem 2007;53:766-772.

[16] Devine J. A review of scrub typhus management in 20002001 and implications for soldiers. J Rural Remote Environ Health 2003;2:14-20.

[17] Hwang TS, Chu YC, Kim YB, Lim BU, Kang JS. Pathologic study of mice infected with Rickettsia tsutsugamushi R19 strain. J Korean Med Sci 1993;8:437-445.

[18] Chrispal A, Boorugu H, Gopinath KG, et al. Scrub typhus: an unrecognized threat in South India - clinical profile and predictors of mortality. Trop Doct 2010;40:129-133.

[19] Charoensak A, Chawalparit O, Suttinont C, et al. Scrub typhus: chest radiographic and clinical findings in 130 Thai patients. J Med Assoc Thai 2006;89:600-607.

[20] Abhilash K, Mannam PR, Rajendran K, John RA, Ramasami P. Chest radiographic manifestations of scrub typhus. $J$ Postgrad Med 2016;62:235-238.

[21] Hwang K, Jang HN, Lee TW, et al. Incidence, risk factors and clinical outcomes of acute kidney injury associated with scrub typhus: a retrospective study of 510 consecutive patients in South Korea (2001-2013). BMJ Open 2017;7: e013882.

[22] Sriwongpan P, Krittigamas P, Kantipong P, Kunyanone N, Patumanond J, Namwongprom S. Clinical indicators for severe prognosis of scrub typhus. Risk Manag Healthc Policy 2013;6:43-49.

[23] Astrup E, Janardhanan J, Otterdal K, et al. Cytokine network in scrub typhus: high levels of interleukin-8 are associated with disease severity and mortality. PLoS Negl Trop Dis 2014;8:e2648. 